\title{
Comparison of the Normal, Preperimetric Glaucoma, and Glaucomatous Eyes with Upper-Hemifield Defects Using SD-OCT
}

\author{
Fusako Fujimura1 ${ }^{*}$, Nobuyuki Shoji ${ }^{1,2}$, Kazunori Hirasawa1, Kazuhiro Matsumura², \\ Tetsuya Morita2 ${ }^{2}$ Kimiya Shimizu² \\ ${ }^{1}$ Department of Rehabilitation, Orthoptics and Visual Science Course, School of Allied Health Sciences, Kitasato \\ University, Tokyo, Japan \\ ${ }^{2}$ Department of Ophthalmology, School of Medicine, Kitasato University, Tokyo, Japan \\ Email: ff-fujimu@kitasto-u.ac.jp, nshoji@ahs.kitasato-u.ac.jp, hirasawa@kitasato-u.ac.jp, \\ kazu-m@dd.iij4u.or.jp, tamosace@gmail.com, ks-secre@kitasato-u.ac.jp
}

Received 15 October 2015; accepted 27 November 2015; published 30 November 2015

Copyright (C 2015 by authors and Scientific Research Publishing Inc.

This work is licensed under the Creative Commons Attribution International License (CC BY). http://creativecommons.org/licenses/by/4.0/

c) (;) Open Access

\begin{abstract}
Purpose: We compared the thickness of circumpapillary retinal nerve fiver layer (cpRNFL) and macular ganglion cell layer with inner plexiform layer (GCL + IPL) using Cirrus HD-OCT (Ver.6.0: Carl Zeiss). Materials and Methods: This study included 12 eyes of normal controls, 10 eyes of preperimetric glaucoma (PPG) with loss of RNFL either in superior or in inferior hemisphere without visual field defects, and 22 eyes of glaucoma eyes with visual field defects restricted to upper hemifield (UHFD: early 10 eyes, severe 12 eyes). The cpRNFL thickness analyzed from disk center by dividing into 12 sectors. The GCL + IPL thickness analyzed from central fovea by dividing into six sectors. Both compared between normal eye group and other 3 groups using the average value of each sectors. Result: The cpRNFL and the GCL + IPL thickness were obviously thin as compared with normal eyes. Conclusion: Even if it is in the state where abnormalities are not detected using the Humphrey field Analyzer, it is suggested that the early structural change of glaucoma has already arisen.
\end{abstract}

\section{Keywords}

Glaucoma, Ganglion Cell Layer, Inner Plexiform Layer, Retinal Nerve Fiver Layer, SD-OCT

${ }^{*}$ Corresponding author.

How to cite this paper: Fujimura, F., Shoji, N., Hirasawa, K., Matsumura, K., Morita, T. and Shimizu, K. (2015) Comparison of the Normal, Preperimetric Glaucoma, and Glaucomatous Eyes with Upper-Hemifield Defects Using SD-OCT. Open Journal of Ophthalmology, 5, 167-173. http://dx.doi.org/10.4236/ojoph.2015.54027 


\section{Introduction}

Glaucoma is an optic neuropathy characterized by loss of retinal ganglion cell (RGC) and thinning of retinal nerve fiber layer (RNFL), and diagnosed by a characteristic alteration of optic nerve head, loss of retinal nerve fiber, defect of visual field. However, when detected abnormal by the funduscopy or visual field testing, $20 \%$ to $40 \%$ of RGC has already disappeared [1] [2]. Earlier studies have shown that the thickness of circumpapillary retinal nerve fiver layer (cpRNFL) begins to decrease before visual field loss [3] [4]. It is difficult to detect the point of disappearance of RGC, or the thinning of RNFL correctly by traditional funduscopy diagnosis and visual field test. There is an issue that a glaucomatous detection is overdue. It is thought required for a glaucomatous early checkup to evaluate quantitatively the structural change of RGC and RNFL. Recently, the spectral domain optical coherent tomography (SD-OCT) is progressing, so it becomes possible to observe and analyze thickness of ganglion cell layer (GCL) and RNFL easily. By using SD-OCT, for glaucomatous eyes, the structure of macula and optic nerve head are examined, and Ganglion Cell Complex (GCC) and the cpRNFL are used as a measurement parameter. The GCC is the sum of RNFL, RGC, inner plexiform layer (IPL) at macular regions. In glaucomatous eyes, the GCC and the cpRNFL are thinner than normal eyes, so, these are useful for the diagnosis of glaucoma [5]-[7]. However, the GCL has been reported to be the early site of glaucomatous damage compared with RNFL and IPL, as shown in experimental model [8]. In the thickness analysis of GCC including RNFL, a possibility that is guessed we miss an early glaucomatous structural change. As opposed to this, the Cirrus HD-OCT (Ver6.0: Carl Zeiss) added the Ganglion Cell Analysis to the existing Glaucoma OU Analysis which was program of the analysis for the cpRNFL. The Ganglion Cell Analysis analyzes the thickness of two layers (GCL + IPL) which excluded RNFL from traditional GCC (RNFL + GCL + IPL). By using this Ganglion cell Analysis, we can consider a possibility to detect an early structural change of glaucoma more sensitively. In this study, we compared the thickness of the cpRNFL and the GCL + IPL using Cirrus HD-OCT (Ver.6.0: Carl Zeiss) in normal eyes, preperimetric glaucoma (PPG) eyes with loss of RNFL in the superior or inferior hemisphere without visual field defects, and glaucoma eyes with visual field defects restricted to the upper hemifield defect (UHFD).

\section{Materials and Methods}

This study protocol adhered to the tenets of the Declaration of Helsinki, and informed consent was obtained from each subjects.

The subjects were 44 eyes of 32 patients (18 eyes of males, 26 eyes of females) who receive regular outpatient treatment for glaucoma or undergo examination for glaucoma suspicion (mean age: $61.2 \pm 10.5$ years, mean equivalent refractive error: $-2.04 \pm 2.65 \mathrm{D}$ ). The visual field was examined by the Humphrey Visual Field Analyzer 740 (Carl Zeiss) using Swedish Interactive Threshold Algorithm 30-2 SITA Standard program. From the obtained result, we judged that their visual fields are normal or abnormal based on the judging standard of Anderson Patella [9]. Moreover, upper visual hemifield defects in glaucomatous eyes were defined that three points of probability symbols were not contiguous in the total deviation plot of the inferior hemifield, and the extent classified based on the Hodapp-Anderson-Parrish classification [7]. Then we divided the subjects into four groups, normal eyes, PPG eyes, early UHFD eyes, severe UHFD eyes. The glaucoma diagnosis was determined by checking glaucomatous defects of optic disk and RNFL with an ophthalmoscopic examination and a fundus photograph, and having a corresponding abnormal visual field. As a result, normal eyes were 12 eyes, PPG eyes were 10 eyes, early UHFD eyes were 10 eyes, severe UHFD eyes were 12 eyes. The age, gender, intra ocular pressure by Goldmann applanation tonometer, spherical equivalent refractive error, mean deviation (MD) value, pattern standard deviation (PSD) value of each group were shown in the Table 1 . There were significant differences in MD value and PSD value among 4 groups ( $<<0.0001$ : one-way ANOVA). There were no differences with age, gender, intraocular pressure, and equivalent refractive error significant among 4 groups (oneway ANOVA, gender: chi-square test). In addition, we gave sufficient explanation about the purport of this research, and after obtaining consent, we performed the following measurement. The cpRNFL and the GCL + IPL thickness measured by Cirrus HD-OCT (Ver.6.0: Carl Zeiss). The $6 \mathrm{~mm}$ square area (200 A-can $\times 200 \mathrm{~A}$-scan) focusing on the optic disk was measured using the Optic Disk Cube $200 \times 200$ scan protocol. The circumference of $1.73 \mathrm{~mm}$ from center of optic nerve disk was divided automatically into 12 sectors using Glaucoma OU Analysis, and the cpRNFL thickness of each sector was analyzed. We used the average value of each sector for examination. In addition, we assigned 12 sectors to the 12th sector clockwise by making into the 1st sector the 1:00 direction which is a clock. The direction of 11, 12 and 1:00 were decided to superior side, the direction of 2, 
Table 1. Background of subjects.

\begin{tabular}{|c|c|c|c|c|c|}
\hline & $\begin{array}{l}\text { Normal eyes } \\
\qquad(n=12)\end{array}$ & $\begin{array}{l}\text { PPG eyes } \\
(n=10)\end{array}$ & $\begin{array}{c}\text { early } \\
\text { UHFD eyes } \\
(\mathbf{n}=10)\end{array}$ & $\begin{array}{c}\text { severe } \\
\text { UHFD eyes } \\
(\mathbf{n}=10)\end{array}$ & $p$-value \\
\hline Age (year) & $64.8 \pm 10.2$ & $55.2 \pm 9.6$ & $61.8 \pm 10.0$ & $59.4 \pm 9.0$ & 0.1453 \\
\hline Gender (M / F) & $4 / 8$ & $4 / 6$ & $5 / 5$ & $5 / 7$ & 0.6330 \\
\hline IOP (mHg) & $15.8 \pm 3.3$ & $16.0 \pm 6.5$ & $14.3 \pm 3.5$ & $13.2 \pm 2.0$ & 0.1608 \\
\hline Equivalent refractive error (D) & $-0.54 \pm 1.48$ & $-2.24 \pm 2.05$ & $-3.30 \pm 3.34$ & $-2.38 \pm 2.51$ & 0.0696 \\
\hline MD value (dB) & $1.45 \pm 0.85$ & $0.63 \pm 1.67$ & $-2.49 \pm 0.80$ & $8.50 \pm 2.60$ & $<0.0001$ \\
\hline PSD value (dB) & $1.68 \pm 0.33$ & $1.80 \pm 0.30$ & $-7.14 \pm 3.35$ & $13.07 \pm 3.56$ & $<0.0001$ \\
\hline
\end{tabular}

PPG eyes: preperimetric glaucoma eyes; UHFD eye: glaucomatous upper-hemifield defect eyes; MD value, PSD value were measured using Humphrey Visual Field Analyzer 740 (Carl Zeiss) using Swedish Interactive Threshold Algorithm 30-2 SITA Standard program one-way ANOVA, gender: chi- square test.

3 and 4:00 were decided to nasal side, the direction of 5, 6 and 7:00 were decided to inferior side, the direction of 8, 9 and 10:00 were decided to temporal side (Figure 1). The $6 \mathrm{~mm}$ square area (200 A-can $\times 200 \mathrm{~A}$-scan) focusing on the macula was measured using the Macular Cube $200 \times 200$ scan protocol. The ellipse of $4.0 \mathrm{~mm}$ long $\times 4.8 \mathrm{~mm}$ side from center of fovea (excluding ellipse of long $1.0 \mathrm{~mm} \times$ wide $1.2 \mathrm{~mm}$ ) was divided automatically into 6 sectors(superior-nasal sector: SN, superior sector: S, superior-temporal sector: ST, inferior-nasal sector: IN, inferior sector: I, inferior-temporal sector: IT) using the Ganglion Cell Analysis, and the GCL + IPL thickness of each sector was analyzed. We used the average value of each of sector for examination (Figure 2). However, in order to be equivalent for UHFD eye, we carry out an up-and-down inversion which is the result of four PPG eyes with RNFL defect in only superior hemisphere, and we added it to six PPG eyes with RNFL defect in only inferior hemisphere, and used it for examination. We compared the cpRNFL thickness and the GCL + IPL thickness between normal eye group and other three groups. Dunnett's test was used statistical analysis, $p$-values less than 0.05 were accepted as statistically significant.

\section{Results}

In inferior hemisphere that was equivalent to visual field defects, the cpRNFL thickness was significantly thinner than normal eyes, the early UHFD eyes were decrease in sector 5, 6, 7 (total 3 sectors), the severe UHFD eyes were decrease in sector 5, 6, 7, 8 (total 4 sector), the PPG eyes were decrease in sector 5, 6, 7 (total 3 sectors). In superior hemisphere that was equivalent to normal visual field, the cpRNFL thickness was significantly thinner than normal eyes, the early UHFD eyes were decrease in sector 11, 12, 1 (total 3 sectors), the severe UHFD eyes were decrease in sector 11, 12, 1, 2 (total 4 sector), the PPG eyes were decrease in sector 1 (Table 2; Figure 3).

In inferior hemisphere, the GCL + IPL thickness was significantly thinner than normal eyes, the early UHFD eyes were decrease in 3 sector (sector IT, I, IN), the severe UHFD eyes were decrease in 3 sector (sector IT, I, IS), the PPG eyes were decrease in 1 sector (sector I). In superior hemisphere, the GCL + IPL thickness was significantly thinner than normal eyes, the early UHFD eyes were decrease in 1 sector (sector SN), the severe UHFD eyes were decrease in 3 sector (sector SN, S, ST), the PPG eyes were decrease in 2 sector (sector SN, ST) (Table 3; Figure 4).

\section{Discussion}

Recently, the examination of RGC and RNFL in glaucoma eye has piled up using remarkable developed OCT. Tan et al. had used the Time-domain OCT and SD-OCT showed that glaucoma leads to thinning of macular nerve fiver, ganglion cell, inner prexiform layers, which was apparent even before visual field changes were detected [10] [11]. Lee et al. had reported that the cpRNFL thickness measured by a SD-OCT was thinner in glaucoma eyes, and the cpRNFL thickness correlated with visual sensitive defects [12]. Moreover, Wu et al. accepted abnormalities by a frequency doubling technology (FDT) in the normal visual field with hemifield defect of glaucoma eye measured by Humphrey Visual Field Analyzer [13]. Takagi et al. reported that the cpRNFL thick- 

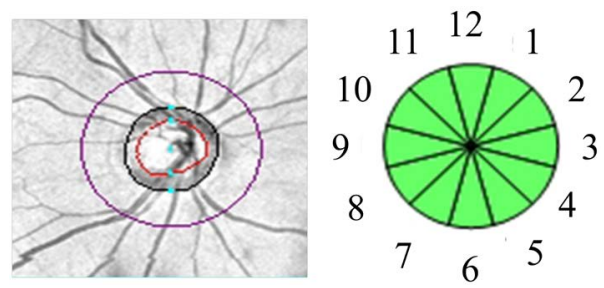

Figure 1. Measurement/Analysis of the cpRNFL. The $6 \mathrm{~mm}$ square area (200 A-can $x$ 200 A-scan) focusing on the optic disk was measured using the Optic Disk Cube $200 \times$ 200 scan protocol. The circumference of $1.73 \mathrm{~mm}$ from center of optic nerve head was divided automatically into 12 sectors using Glaucoma OU Analysis, and the cpRNFL thickness of each sector was analyzed. We assigned 12 sectors to the 12th sector clockwise by making into the 1st sector the 1:00 direction which is a clock. The direction of 11,12 and 1:00 were decided to superior side, the direction of 2, 3 and 4:00 were decided to nasal side, the direction of 5, 6 and 7:00 were decided to inferior side, the direction of 8, 9 and 10:00 were decided to temporal side.
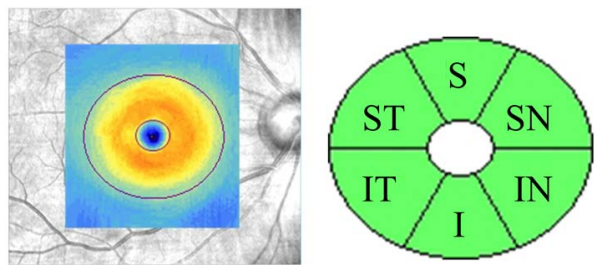

Figure 2. Measurement/Analysis of the GCL + IPL. The $6 \mathrm{~mm}$ square area (200 A-can $\times$ 200 A-scan) focusing on the macula was measured using the Macular Cube $200 \times 200$ scan protocol. The ellipse of $4.0 \mathrm{~mm}$ long $\times 4.8 \mathrm{~mm}$ side from center of fovea (excluding ellipse of long $1.0 \mathrm{~mm} \times$ wide $1.2 \mathrm{~mm}$ ) was divided automatically into 6 sectors (superior-nasal side: SN, superior side: S, superior-temporal side: ST, inferior-nasal side: IN, inferior side: I, inferior-temporal side: IT) using Ganglion Cell Analysis, and the GCL + IPL thickness of each sector was analyzed.

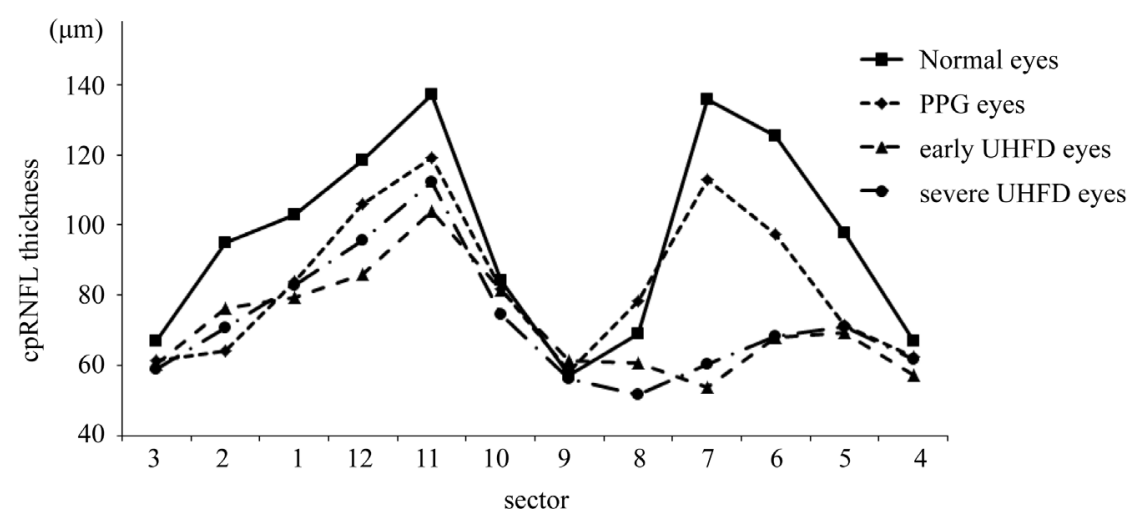

Figure 3. Results or the cpRNFL thickness. In inferior hemisphere that was equivalent to visual field defects, the cpRNFL thickness was significantly thinner than normal eyes, the early UHFD eyes were decrease in 3 sectors, the severe UHFD eyes were decrease in 4 sectors, the PPG eyes were decrease in 3 sectors. In superior hemisphere that was equivalent to normal visual field, the cpRNFL thickness was significantly thinner than normal eyes, the early UHFD eyes were decrease in 3 sectors, the severe UHFD eyes were decrease in 4 sectors, the PPG eyes were decrease in 1 sector. The results of statistical analysis were shown in Table 2.

ness, the GCC (RNFL + GCL + IPL) thickness of normal and abnormal hemisphere with the glaucomatous hemifield visual defect were thinner than normal eyes [5]. For these reasons, the normal hemifield with glaucomatous hemifeild defect measured by a static perimetry was regarded as a preceding state which glaucomatous 
Table 2. Results of the cpRNFL thickness $(\mu \mathrm{m})$.

\begin{tabular}{cccccc}
\hline sector & & Normal eyes & PPG eyes & early UHFD eyes & severe UHFD eyes \\
\hline \multirow{2}{*}{ superior } & 11 & $137.1 \pm 25.9$ & $119.2 \pm 22.7$ & $103.8 \pm 14.2^{* *}$ & $112.3 \pm 24.4^{*}$ \\
& 12 & $118.6 \pm 18.9$ & $106.0 \pm 14.3$ & $85.8 \pm 24.4^{*}$ & $95.8 \pm 23.9^{* *}$ \\
& 1 & $102.8 \pm 14.4$ & $83.7 \pm 11.0^{*}$ & $79.2 \pm 15.6^{* *}$ & $82.8 \pm 18.5^{*}$ \\
& 2 & $94.8 \pm 26.4$ & $64.0 \pm 10.7$ & $76.1 \pm 25.4^{* *}$ & $70.6 \pm 11.4^{* *}$ \\
nasal & 3 & $66.8 \pm 9.7$ & $61.3 \pm 5.5$ & $60.4 \pm 18.1$ & $59.0 \pm 10.1$ \\
& 4 & $66.8 \pm 7.1$ & $62.4 \pm 7.3$ & $57.2 \pm 12.1$ & $61.6 \pm 10.8$ \\
inferior & 5 & $97.8 \pm 21.7$ & $71.5 \pm 18.2^{* *}$ & $69.3 \pm 19.5^{* *}$ & $70.9 \pm 9.4^{* *}$ \\
& 6 & $125.3 \pm 25.1$ & $97.5 \pm 17.0^{* *}$ & $67.9 \pm 13.6^{* * *}$ & $68.4 \pm 18.4^{* * *}$ \\
& 7 & $135.8 \pm 23.7$ & $112.8 \pm 25.5^{*}$ & $53.6 \pm 14.8^{* * *}$ & $60.3 \pm 9.8^{* * *}$ \\
& 8 & $68.8 \pm 12.3$ & $78.5 \pm 19.5$ & $60.5 \pm 11.3$ & $51.8 \pm 10.7^{* *}$ \\
temporal & 9 & $57.2 \pm 11.3$ & $58.2 \pm 9.8$ & $61.2 \pm 6.9$ & $56.3 \pm 12.3$ \\
& 10 & $84.3 \pm 17.9$ & $81.8 \pm 18.3$ & $81.3 \pm 12.3$ & $74.6 \pm 13.3$ \\
\hline
\end{tabular}

PPG eyes: preperimetric glaucoma eyes; UHFD eye: glaucomatous upper-hemifield defect eyes; *: p < 0.05; **: p < 0.01; ***: p < 0.001, Dunnett’s test.

Table 3. Result of the GCL + IPL thickness $(\mu \mathrm{m})$.

\begin{tabular}{ccccc}
\hline sector & Normal eyes & PPG eyes & early UHFD eyes & severe UHFD eyes \\
SN & $85.6 \pm 7.8$ & $76.8 \pm 4.6^{*}$ & $75.2 \pm 10.0^{*}$ & $76.2 \pm 8.7^{*}$ \\
S & $84.0 \pm 6.7$ & $76.2 \pm 3.1$ & $73.8 \pm 14.7$ & $71.2 \pm 14.6^{*}$ \\
ST & $81.9 \pm 6.3$ & $74.3 \pm 5.3^{* *}$ & $76.1 \pm 5.9$ & $71.3 \pm 5.2^{* * *}$ \\
IT & $82.3 \pm 6.7$ & $76.9 \pm 5.8$ & $58.6 \pm 6.4^{* * *}$ & $54.8 \pm 4.0^{* * *}$ \\
I & $81.3 \pm 5.7$ & $73.2 \pm 5.6^{*}$ & $66.3 \pm 8.9^{* * *}$ & $57.9 \pm 5.2^{* * *}$ \\
IN & $82.8 \pm 6.7$ & $75.0 \pm 4.2$ & $71.0 \pm 11.6^{*}$ & $69.3 \pm 8.3^{* * *}$ \\
\hline
\end{tabular}

*: $\mathrm{p}<0.05 ; * *: \mathrm{p}<0.01 ; * * *: \mathrm{p}<0.001$; Dunnett's test superior-nasal side: SN, superior side: S, superior-temporal side: ST, inferior-nasal side: IN, inferior side: I, inferior-temporal side: IT.

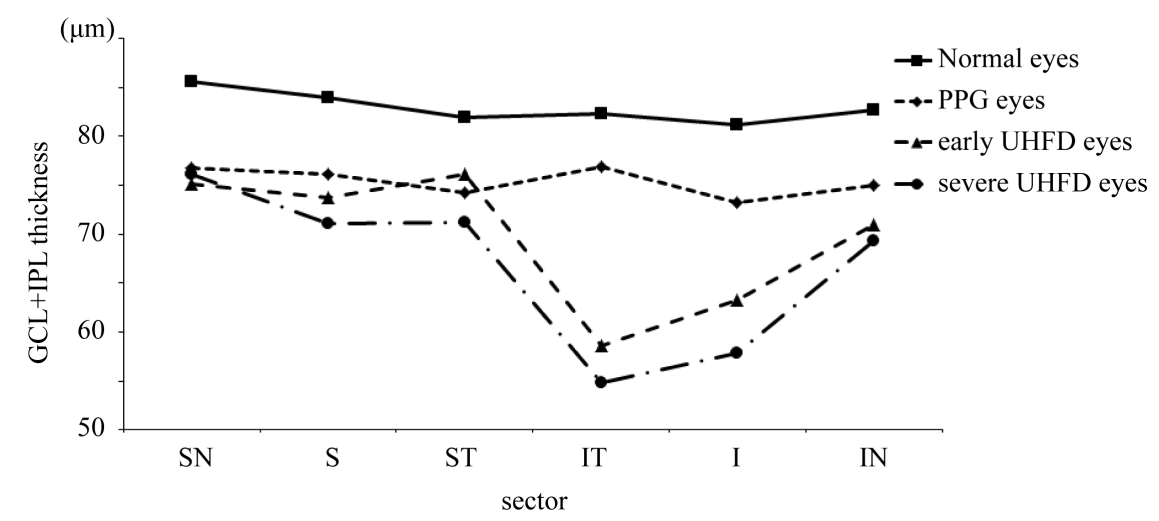

Figure 4. Results of the GCL + IPL thickness. In inferior hemisphere, the GCL + IPL thickness was significantly thinner than normal eyes, both the early and the severe UHFD eyes were decrease in 3 sectors, the PPG eyes were decrease in 1 sector. In superior hemisphere, the GCL + IPL thickness was significantly thinner than normal eyes, the early UHFD eyes were decrease in 1 sector, the severe UHFD eyes were decrease in 3 sectors, the PPG eyes were decrease in 2 sectors. The results of statistical analysis were shown in Table 3. 
visual field change produces.

In this study, we compared the thickness of the cpRNFL and the GCL + IPL using Cirrus HD-OCT (Ver.6.0: Carl Zeiss) in normal eyes, PPG eyes, UHFD eyes (early and severe). As a result, also not only in the abnormality side but the normal side of UHFD eyes, the thickness of the cPRNFL and the GCL + IPL were significantly less than in normal eyes, we consider that it was able to detect that the glaucomatous structural change has arisen in the normal visual field with a glaucomatous visual hemifield defect the same as the past reports. In addition, in this study, the cpRNFL thickness and the GCL + IPL thickness of PPG eyes were significant thinner than normal eye, so it is suggested that in the state with the RNFL deficit, the structural change of glaucoma has developed the same as UHFD eye, even if there are no abnormalities in the result of the Humphrey Visual Field Analyzer. Considering the circumstances mentioned above, it is suggested that the result of measurement by the SD-OCT can be one of the important diagnostic views in progress observation of not only glaucoma eyes but also PPG eyes.

In the past report using the SD-OCT, the GCC which is the sum of the thickness of macular RNFL, GCL, IPL was adopted. Anatomically, the human retina contain more than 1 million RGCs, with substantial inter individual variability; approximately $50 \%$ of the cell are concentrated within $4.5 \mathrm{~mm}$ of fovea, and there is no variability in the RGC population in this small parafoveal area than the RNFL population [14]. Furthermore, the axons of not only macular RGCs but also those of some peripheral RGCs outside of macular pass through the macular area to reach optic nerve head, so it is difficult to separate and evaluate the change of macular RGC and a circumference part from the cpRNFL thickness. Moreover, in the recent reports, the cpRNFL thickness was influenced by age, ethnicity, axial length, optic disc size, and it was obviously that the result of the cpRNFL thickness has brought errors [15] [16]. It is suggested that the quantitative evaluation of the macular structure is the more sensitive method to detect a structure changes of the early glaucoma. However, the GCL has been reported to be the early site of glaucomatous damage, as shown in experimental models [7]. In the past examinations, it is guessed the macular GCC including the RNFL is without high ability to detect a glaucomatous structural change.

The Ganglion Cell Analysis in the Cirrus HD-OCT is the program to analyze only two layers thickness (GCL and IPL) excluding RNFL. Compared with evaluation of GCC, it is thought that the GCL + IPL analysis (Ganglion cell Analysis) has potential to be useful for glaucoma early detection. Mwanza et al. reported that the ability of macular GCIPL parameters to discriminate normal eyes and early glaucoma eyes is high and comparable to that of the best cpRNFL and ocular nerve head parameters [17]. In this study, we have not carried out comparison of the power to detect of the GCL + IPL, and the GCC (RNFL + GCL + IPL) and the cpRNFL. It needs future developments.

\section{Conclusion}

In this study, we compared the thickness of the cpRNFL and the GCL + IPL using Cirrus HD-OCT (Ver.6.0: Carl Zeiss) in normal eyes, PPG eyes, UHFD eyes. As a result, not only in the abnormality side of UHFD eyes but also in the normal side of UHFD eyes and PPG eyes, the thickness of the cPRNFL and the GCL + IPL were significantly less than in normal eyes. It is suggested that in the state with RNFL deficit, the structural change of glaucoma has developed, even if there are no abnormalities in the result of the Humphrey Visual Field Analyzer.

\section{Institutional Review Board}

This study was approved by the Institutional Review Board at Kitasato University Hospital (number B13-152, 2013) and followed the tenets of the Declaration of Helsinki.

\section{Acknowledgements}

The authors report no conflicts of interest. The authors alone are responsible for the content and writing of the paper.

\section{References}

[1] Quigley, H.A., Dunkelberger, G.R. and Green, W.R. (1989) Retinal Ganglion Cell Atrophy Correlated with Automated Perimetry in Human Eyes with Glaucoma. American Journal of Ophthalmology, 107, 453-464. 
[2] Kerrigan-Baumrind, L.A., Quigley, H.A., Pease, M.E., et al. (2000) Number of Ganglion Cells in Glaucoma Eyes Compared with Threshold Visual Field Tests in the Same Persons. Investigative Ophthalmology \& Visual Science, 41, 741-748.

[3] Kanamori, A., Nakamura, M., Escano, M.F., et al. (2003) Evaluation of the Glaucomatous Damage on Retinal Nerve Fiber Layer Thickness Measured by Optical Coherence Tomography. American Journal of Ophthalmology, 135, 513-520. http://dx.doi.org/10.1016/S0002-9394(02)02003-2

[4] Wollstein, G., Ishikawa, H., Wang, J., et al. (2005) Comparison of Three Optical Coherence Tomography Scanning Areas for Detection of Glaucomatous Damage. American Journal of Ophthalmology, 139, 39-43. http://dx.doi.org/10.1016/j.ajo.2004.08.036

[5] Takagi, S.T., Kita, Y., Yagi, F., et al. (2012) Macular Retinal Ganglion Cell Complex Damage in the Apparently Normal Visual Field of Glaucomatous Eyes with Hemifield Defects. Journal of Glaucoma, 21, 318-325. http://dx.doi.org/10.1097/IJG.0b013e31820d7e9d

[6] Rao, H.L., Zangwill, L.M., Weinreb, R.N. et al. (2010) Comparison of Different Spectral Domain Optical Coherence Tomography Scanning Areas for Glaucoma Diagnosis. Ophthalmology, 117, 1692-1699.

[7] Huang, J.Y., Pekmezci, M., Mesiwala, N., et al. (2010) Diagnostic Power of Optic Disc Morphology, Peripapillary Retinal Nerve Fiber Layer Thickness, and Macular Inner Retinal Layer Thickness in Glaucoma Diagnosis with Fourier-Domain Optical Coherence Tomography. Journal of Glaucoma, 20, 87-94. http://dx.doi.org/10.1097/IJG.0b013e3181d787b6

[8] Desatnik, H., Quigley, H.A. and Glovinsky, Y. (1996) Study of Central Retinal Ganglion Cell Loss in Experimental Glaucoma in Monkey Eyes. Journal of Glaucoma, 5, 46-53.

[9] Anderson, D.R. and Patella, V.M. (1990) Automated Static Perimetry. $2^{\text {nd }}$ Edition, St. Louis: Mosby, 121-190.

[10] Tan, O., Li, G., Lu, A.T., et al. (2008) Mapping of Macular Substructures with Optical Coherence Tomography for Glaucoma Diagnosis. Ophthalmology, 115, 949-956.

[11] Tan, O., Chopra, V., Lu, A.T., et al. (2009) Detection of Macular Ganglion Cell Loss in Glaucoma by Fourier-Domain Optical Coherence Tomography. Ophthalmology, 116, 2305-2314.

[12] Lee, J.R., Jeoung, J.W., Choi, J., et al. (2010) Structure-Function Relationships in Normal and Glaucomatous Eyes Determined by Time Domain and Spectral Domain Optical Coherence Tomography. Investigative Ophthalmology \& Visual Science, 51, 6424-6430. http://dx.doi.org/10.1167/iovs.09-5130

[13] Wu, L.L., Suzuki, Y., Kunimatsu, S., et al. (2001) Frequency Doubling Technology and Confocal Scanning Ophthalmoscopic Optic Disc Analysis in Open-Angle Glaucoma with Hemifield Defects. Journal of Glaucoma, 10, $256-260$. http://dx.doi.org/10.1097/00061198-200108000-00002

[14] Curcio, C.A. and Allen, K.A. (1990) Topography of Ganglion Cells in Human Retina. Journal of Comparative Neurology, 300, 5-25. http://dx.doi.org/10.1002/cne.903000103

[15] Bendschneider, D., Tornow, R.P., Horn, F.K., et al. (2010) Retinal Nerve Fiber Layer Thickness in Normals Measured by Spectral Domain OCT. Journal of Glaucoma, 19, 475-482. http://dx.doi.org/10.1097/IJG.0b013e3181c4b0c7

[16] Budenz, D.L., Anderson, D.R., Varma, R., et al. (2007) Determinants of Normal Retinal Nerve Fiber Layer Thickness Measured by Stratus OCT. Ophthalmology, 114, 1046-1052. http://dx.doi.org/10.1016/j.ophtha.2006.08.046

[17] Mwanza, J.C., Durbin, M.K., Budenz, D.L., et al. (2012) Glaucoma Diagnostic Accuracy of Ganglion Cell-Inner Plexiform Layer Thickness: Comparison with Nerve Fiber Layer and Optic Nerve Head. Ophthalmology, 119, 1151-1158. http://dx.doi.org/10.1016/j.ophtha.2011.12.014 